\title{
Dynamics of adipose tissue turnover in human metabolic health and disease
}

\author{
Ursula White $^{1}$ (D) Eric Ravussin ${ }^{2}$
}

Received: 10 May 2018 / Accepted: 25 July 2018 /Published online: 28 September 2018

(C) Springer-Verlag GmbH Germany, part of Springer Nature 2018

\begin{abstract}
White adipose tissue is a highly plastic organ and is an important regulator of whole-body metabolism and energy balance. The magnitude of adipose tissue mass is determined by dynamic changes in the synthesis and breakdown (i.e. turnover) of adipocytes and triacylglycerols (TGs). Obesity is a disorder characterised by excessive adiposity and is a risk factor for diseases, including the metabolic syndrome and type 2 diabetes. Adipose tissue expansion is necessary to accommodate chronic excess energy intake and is characterised by enlargement of existing adipocytes (hypertrophy) and by increase in pre-adipocyte and adipocyte numbers (hyperplasia). Evidence suggests that the manner of subcutaneous adipose expansion can influence metabolic health, as impaired adipogenesis, namely restricted hyperplasia, may lead to ectopic lipid deposition in the liver and skeletal muscle, contributing to the pathogenesis of obesity-related disorders. Despite the plausible role of adipose turnover in human health and pathology, little is known about the in vivo kinetics of adipose tissue components (both adipose cells and TGs). This is due, in part, to the slow turnover rate of adipose tissue and the complexity of directly labelling pathway precursors. This review provides a brief summary of findings derived from in vitro techniques, as well as an overview of two in vivo methods that are being implemented to assess the turnover of adipose cells and TGs. Finally, the role of adipose tissue turnover in metabolic health and disease is discussed.
\end{abstract}

Keywords Adipocyte - Adipocyte death · Adipose kinetics - Adipose lipid turnover - Adipose tissue $\cdot$ Metabolic disease Metabolic health $\cdot$ Obesity $\cdot$ Review $\cdot$ Triacylglycerol

$\begin{array}{ll}\text { Abbreviations } \\ \text { BAT } & \text { Brown adipose tissue } \\ \text { MIDA } & \text { Mass isotopomer distribution analysis } \\ \text { scABD } & \text { Subcutaneous abdominal } \\ \text { scFEM } & \text { Subcutaneous femoral } \\ \text { TG } & \text { Triacylglycerol } \\ \text { VAT } & \text { Visceral adipose tissue } \\ \text { WAT } & \text { White adipose tissue }\end{array}$

Electronic supplementary material The online version of this article (https://doi.org/10.1007/s00125-018-4732-x) contains a slide of the figure for download, which is available to authorised users.

Ursula White

ursula.white@pbrc.edu

1 Physiology of Human Adipose Tissue Laboratory, Pennington Biomedical Research Center, Louisiana State University System, Baton Rouge, LA 70808, USA

2 Human Translational Physiology Laboratory, Pennington Biomedical Research Center, Louisiana State University System, Baton Rouge, LA, USA

\section{Introduction}

Adipose tissue is an essential organ and is the primary site for energy storage as triacylglycerols (TGs). White adipose tissue (WAT) and brown adipose tissue (BAT) are the predominant types in mammals. As WAT and BAT have different developmental origins and the majority of human adipose tissue is WAT, this type will be the focus of this review. Traditionally, studies of WAT function have revolved around its significant role in controlling energy homeostasis by storing and releasing lipids in response to energy demands. However, WAT has additional properties, including secretory functions (release of adipokines), that affect insulin sensitivity and metabolic homeostasis, contributing to the pathogenesis of obesity and related comorbidities [1].

WAT is distributed throughout the body in several depots, each with distinct functions. Evidence suggests that regional distribution of WAT, rather than overall obesity, may be a stronger predictor of metabolic health risks. The accumulation of fat in the upper body (namely visceral adipose tissue [VAT] but also subcutaneous abdominal 
[scABD]) confers a higher risk of developing type 2 diabetes mellitus and cardiovascular disease, while in the lower body, subcutaneous gluteal and subcutaneous femoral (scFEM) fat may be metabolically protective [2]. Sexual dimorphism in fat distribution is also well-documented: women, despite having, on average, a higher percentage of body fat than men, display preferential adipose accumulation in the 'protective' lower-body depots [3]. Conversely, men tend to deposit more fat in the upperbody VAT depot [4]. Sex differences in fat distribution and the distinct functions of adipose depots have been discussed in previous reviews $[5,6]$.

Although most adipose growth occurs during childhood and adolescence, WAT retains the ability to expand during adulthood in response to fluctuations in energy balance. The magnitude of adiposity during the development of obesity is determined by dynamic changes in the synthesis and removal (i.e. turnover) of adipose cells (pre-adipocytes and adipocytes) and TGs. Despite the negative health consequences associated with obesity, little is known about in vivo WAT turnover in humans. This review provides a brief summary of findings obtained from in vitro methods, as well as an overview of two in vivo approaches that are being implemented to assess the turnover of adipose cells and TGs. Finally, the role of WAT turnover in metabolic health and disease is discussed.

\section{Adipose turnover}

\section{Adipose expansion}

WAT stores energy in the form of TGs and releases fatty acids via lipolysis to maintain metabolic homeostasis. To accommodate lipids, adipose expansion occurs via the enlargement of existing adipocytes (hypertrophy) and/or the proliferation and differentiation of pre-adipocytes to adipocytes (hyperplasia or adipogenesis). Evidence suggests that the manner of subcutaneous WAT expansion (hypertrophy vs hyperplasia) in humans can influence cardiometabolic health. Cross-sectional and longitudinal studies have shown that hypertrophic abdominal adipocytes are associated with insulin resistance [7, 8] and type 2 diabetes $[9,10]$, independent of overall obesity. It has also been proposed that impaired adipogenesis, or restricted hyperplasia, may lead to ectopic fat deposition in the liver and skeletal muscle, contributing to the pathogenesis of obesity-related disorders [11]. Arner et al developed an 'adipocyte morphology value', defined as the difference between the measured adipocyte size and the expected size given the curve-linear fit for a given body mass, to predict scABD cellularity in humans [12]. These authors reported that greater scABD hypertrophy (i.e. higher morphology index) was linked to insulin resistance and lower adipocyte number, independent of sex and adiposity, while greater hyperplastic expansion was associated with better insulin sensitivity. Collectively, the above-mentioned data support the 'adipose tissue expandability hypothesis', which postulates that a lack of hyperplasia, coupled with a high prevalence of hypertrophic adipocytes, results in a limited capacity of WAT to expand and store fat, thus causing impaired metabolic health [13].

However, the role of adipogenesis (i.e. hyperplasia) in health risks is debatable. Recent observations reported a higher prevalence of small fat cells in insulin-resistant individuals and in those with type 2 diabetes, compared with healthy individuals, suggesting hyperplastic expansion [8, 14-16]. Importantly, we showed that 8 weeks of $40 \%$ overfeeding ( $\sim 7.6 \mathrm{~kg}$ weight gain) resulted in greater impairment of insulin sensitivity in individuals who had smaller adipocytes at baseline compared with those who had larger adipocytes [17]. Another human overfeeding study demonstrated that smaller adipocyte size at baseline predicted a decline in insulin sensitivity following a $3.2 \mathrm{~kg}$ weight gain over 4 weeks [18]. These recent studies are contrary to the adipose expandability hypothesis and indicate that an increased population of small adipocytes (suggesting hyperplasia) is associated with impaired metabolic health outcomes.

Depot differences in adipose expansion The opposing associations of upper-body vs lower-body fat distribution with metabolic health are probably due to intrinsic characteristics of WAT depots. Few studies have assessed regional differences in adipogenesis in humans. Depot differences in preadipocytes cultured from WAT have been reported, with scABD fat having higher proliferative and differentiation capacity than scFEM fat $[19,20]$ and VAT [21]. Another adipocyte size assessment suggested depot-specific fat expansion in response to overfeeding: mainly hypertrophy in scABD fat and primarily hyperplasia in scFEM fat [22].

Sex differences in adipose expansion Studies in the 1980s reported hyperplastic expansion (higher adipocyte number) of lower-body depots in women and abdominal depots in men [23]. More recent analyses of fat cell size suggest that lower-body adipose tissue expansion is primarily governed by hyperplasia in women but by hypertrophy in men [24]. A higher percentage of early differentiated adipocytes has been identified in the scFEM depot of women relative to men [20].

\section{Adipocyte death}

In addition to adipose expansion, adipocyte death is a vital component of adipose turnover [25]. Rodent studies reveal an oscillatory pattern of adipose cellularity, including cycles of hypertrophy, hyperplasia and adipocyte death, with increased adipocyte death in obese rodents $[25,26]$. Data in humans are scarce. Increased adipocyte death has been implicated in human obesity [25]. Increased response to apoptotic 
stimuli of VAT (vs scABD fat) pre-adipocytes has been reported $[27,28]$. One study suggests that scABD pre-adipocytes from obese women are more susceptible to apoptosis than the scFEM pre-adipocytes [20]. To date, no studies have assessed in vivo adipocyte death in humans and its functional link to fat distribution and metabolic health.

\section{In vivo studies to measure adipose turnover}

As highlighted above, established in vitro methods have been employed to assess adipose cellularity and turnover and have yielded valuable information. Primary cultures, as well as functional assays and expression analyses of genes and proteins, have been commonly used to investigate mechanisms of adipogenesis [21]. A standard approach to estimate adipocyte cellularity and lipid content is to determine fat cell size via microscopy or osmium tetroxide fixation [14]. Other assessments in rodents have estimated adipocyte size distributions via microbiopsies of WAT collected at different time points to assess adipocyte cellularity with weight gain [26]. Although in vitro and indirect approaches provide insight into the mechanisms of adipose expansion and adipocyte death, these methods do not provide an assessment in the WAT's natural milieu. Hence, in vivo approaches are necessary to assess adipose turnover accurately. Nevertheless, the assessment of fat kinetics in vivo has been very difficult in humans, due in part to the slow turnover rate of WAT and the complexity of directly labelling pathway precursors. Although informative in rodents, the incorporation of labelled nucleotides, such as ${ }^{3} \mathrm{H}$-labelled thymidine or 5-bromodeoxyuridine, involves several limitations, including toxicity and biochemical complications, and thus is not applicable for use in humans. Therefore, despite the broad acceptance of the adipose expandability hypothesis, there is a scarcity of data to describe the in vivo dynamics of adipose turnover and the link with metabolic health in humans. Early studies proposed that adipocytes are produced only during childhood and adolescence [29] but in vivo evidence is necessary to substantiate adipocyte generation in adulthood. Below, we review two novel and distinct in vivo approaches that have been implemented in humans.

\section{${ }^{2} \mathrm{H}$ labelling method}

A novel method was developed by Hellerstein's laboratory to assess in vivo adipose kinetics using the incorporation of the stable isotope deuterium into adipose tissue [30]. The protocol, which was validated in mice and then in humans, includes an initial ramp-up of ${ }^{2} \mathrm{H}_{2} \mathrm{O}$ (heavy water) to achieve near-plateau systemic ${ }^{2} \mathrm{H}$ enrichment of body water, followed by sufficient daily intake to maintain enrichment values [30] (Fig. 1). The ${ }^{2} \mathrm{H}$ from the heavy water is then incorporated into adipose cells, and

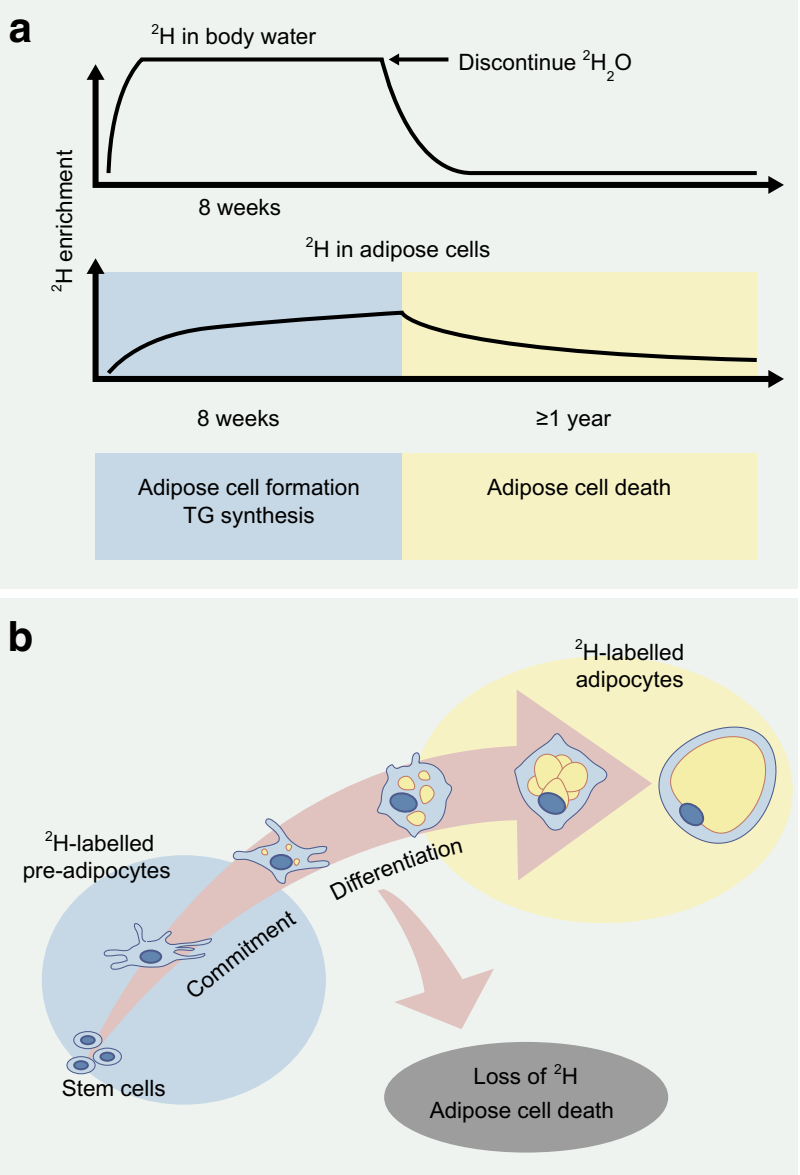

Fig. $1{ }^{2} \mathrm{H}$ labelling protocol to assess in vivo adipose turnover. (a) The deuterium labelling method has been validated to provide in vivo estimates of adipose cell formation, TG synthesis and adipocyte death in humans via the incorporation of the stable isotope ${ }^{2} \mathrm{H}$ into body water and adipose tissue. ${ }^{2} \mathrm{H}$ is administered $\mathrm{as}^{2} \mathrm{H}_{2} \mathrm{O}$ in such a way as to achieve near-plateau systemic ${ }^{2} \mathrm{H}$ enrichment of body water, followed by daily intake to maintain enrichment values. Isotope enrichment of adipose cells is measured via mass spectrometry and application of MIDA. (b) The enrichment of ${ }^{2} \mathrm{H}$ into adipose tissue (pre-adipocytes and adipocytes) provides measures of adipose cell formation and death. This figure is available as a downloadable slide

isotope enrichment is measured via mass spectrometry and application of mass isotopomer distribution analysis (MIDA). Measures of ${ }^{2} \mathrm{H}$-labelled DNA synthesis denote newly formed pre-adipocytes and adipocytes (via pre-adipocyte differentiation) [31], while ${ }^{2} \mathrm{H}$ incorporation in the glycerol moiety represents TG synthesis [32].

Hellerstein and colleagues applied the ${ }^{2} \mathrm{H}$-labelling approach to provide in vivo evidence that adipocytes are generated in humans during adulthood [33]. We have recently assessed in vivo kinetics in subcutaneous WAT from the scABD and scFEM depots using an 8 week incorporation of ${ }^{2} \mathrm{H}$ into adipose cells in obese women [34]. We reported, for the first time, depot differences in in vivo kinetics, with higher adipose cell formation (pre-adipocytes and adipocytes) and TG synthesis rates in the 
scFEM depot relative to the scABD depot $[34,38]$. These data demonstrate that in vivo adipose cell kinetics differ among WAT depots in humans and strengthen other experimental observations that implicate the thigh as a more efficient depot to expand and accommodate lipids in women [2,5]. Interestingly, adipose cell formation rates in both scABD and scFEM depots were positively correlated with percentage body fat, BMI and visceral adiposity, while negatively associated with insulin sensitivity [35]. These novel data challenge the adipose tissue expandability hypothesis and provide the first evidence of an association between facets of impaired metabolic health and higher, not lower, in vivo adipose cell formation.

Hellerstein's laboratory was also the first to apply the ${ }^{2} \mathrm{H}$ labelling method to directly measure TG synthesis and turnover in human WAT in vivo [33]. TG half-life was determined to be $\sim 6$ months [33], similar to previous estimates made by indirect techniques [36]. Other laboratories have applied the ${ }^{2} \mathrm{H}$ labelling approach to demonstrate that TG kinetics in WAT plays a significant role in metabolism, reporting that in vivo rates of TG synthesis were significantly lower in insulinresistant individuals than in insulin-sensitive individuals [37]. Interestingly, we recently identified race and depot differences in TG replacement, with a higher rate of TG synthesis in white vs black individuals and in the scFEM vs scABD depot [38]. These data are intriguing, as race differences in cardiometabolic health have been described [39].

\section{${ }^{14} \mathrm{C}$ dating method}

In vivo adipocyte turnover in humans has also been estimated by measuring the integration of atmospheric ${ }^{14} \mathrm{C}$, released from nuclear bomb tests during the Cold War (1955-1963), into the genomic DNA of WAT [40]. After cessation of the nuclear bomb testing period, the levels of ${ }^{14} \mathrm{C}$ in the environment exponentially decreased, and ${ }^{14} \mathrm{C}$ was incorporated into plants. The consumption of plants, or animals having eaten plants, resulted in ${ }^{14} \mathrm{C}$ concentrations in the human body reflecting those in the atmosphere at a given time. Hence, this retrospective analysis estimated net changes in adipose turnover over a period of approximately four decades by measuring ${ }^{14} \mathrm{C}$ levels in DNA via accelerator mass spectrometry and relating these values to ${ }^{14} \mathrm{C}$ atmospheric data.

Spalding et al reported that $\sim 8.4 \%$ of scABD adipocytes in vivo are replaced every year, regardless of age or degree of obesity [40]. The authors also suggested that adipocyte generation and death rates were in equilibrium in individuals with early onset obesity and that total fat cell number is tightly regulated, even with weight gain or loss. These data suggest that total adipocyte number may be set during childhood and adolescence and constant during adulthood. However, additional studies are necessary to assess fat turnover in individuals with onset of obesity later in life who gradually gain weight over a prolonged period of time, as changes in total adipocyte number may occur. Indeed, one study has shown that total adipocyte number increased in the scFEM depot of women during overfeeding [22]. As in vitro data based on the adipocyte morphology index supports the adipose tissue expandability hypothesis (discussed above), Arner et al observed the relationship of the morphology values to the in vivo generation of adipocytes [12]. Interestingly, individuals displaying more hypertrophic fat expansion (higher morphology index) produced fewer adipocytes in vivo per year than individuals displaying hyperplasia.

Spalding and colleagues also applied the ${ }^{14} \mathrm{C}$ dating method to study long-term in vivo WAT lipid turnover. The mean age of scABD adipocyte TGs, reflected as the duration of TG storage in WAT until irreversible removal via lipolysis (i.e. TG lifespan), was reported to be $\sim 1.6$ years [41]. Because the authors estimated adipocyte age as $\sim 9.5$ years, this implies that TGs are replaced roughly six times during the adipocyte lifespan. Lower TG age was significantly associated with higher in vitro adipocyte lipolysis (stimulated), implicating lipolysis as an important determinant of TG removal. Interestingly, there was no difference in TG age between sexes or among adipocyte sizes. Net TG storage was calculated to represent the amount of TG stored in WAT each year. Obese individuals had higher TG storage and TG age relative to nonobese individuals, implying that high storage and low removal of adipose TGs may be determinants of obesity [41]. Interestingly, mean TG age, but not TG storage, was correlated with insulin resistance, as assessed by HOMA-IR. Overall, Spalding and colleagues propose that the storage and removal of lipids (TGs), similar to adipocyte turnover, is constant during adulthood. Nevertheless, the lack of variation in TG age as assessed long term does not discount the presence of shortterm changes in the dynamics of lipid turnover.

\section{Perspectives on in vivo approaches to measure adipose turnover}

The aforementioned in vivo data refute the previous hypothesis that adipocyte formation is limited to childhood and adolescence and validates the notion that humans generate adipose cells in various depots during adulthood $[34,40]$. Collectively, these methods are a substantive departure from prior in vitro approaches and provide the first novel insight into in vivo adipose turnover in humans (Table 1). Nevertheless, there are some considerations to be addressed. It is important to note that the ${ }^{14} \mathrm{C}$ dating and ${ }^{2} \mathrm{H}$ labelling methods are very different protocols. The assessment of 'naturally' occurring ${ }^{14} \mathrm{C}$ enrichment was a retrospective analysis to assess long-term turnover. This approach may underestimate turnover, as adipocytes derived from the differentiation of post-mitotic pre-adipocytes may not be integrated with ${ }^{14} \mathrm{C}$. Furthermore, some labelled adipocytes may not be captured for analysis because of their 
Table 1 Summary of findings from the ${ }^{2} \mathrm{H}$ and ${ }^{14} \mathrm{C}$ in vivo methods

\begin{tabular}{|c|c|c|}
\hline Variable & ${ }^{2} \mathrm{H}$ method & ${ }^{14} \mathrm{C}$ method \\
\hline Study duration & Short term ( $\sim 8$ weeks $)$ & $\begin{array}{l}\text { Long term } \\
\text { Retrospective ( } \sim 40 \text { years) }\end{array}$ \\
\hline \multicolumn{3}{|l|}{ Study population } \\
\hline Sex & Male and female & Male and female \\
\hline Obesity status & Obese & Lean and obese \\
\hline Adipose samples & scABD and scFEM & $\mathrm{scABD}$ \\
\hline Adipocyte formation & $\uparrow$ in scFEM vs scABD [34] & $\begin{array}{l}\text { Estimated to be equal to adipocyte } \\
\text { deaths }(\sim 8.4 \% / \text { year) }[40]\end{array}$ \\
\hline Adipocyte death & Not measured & $\sim 8.4 \%$ adipocyte death/year [40] \\
\hline $\begin{array}{l}\text { Associations between } \\
\text { adipocyte formation } \\
\text { vs metabolic health }\end{array}$ & $\begin{array}{l}+\% \text { Body fat and BMI } \\
+ \text { Visceral adiposity } \\
- \text { Insulin sensitivity } \\
{[35]^{\mathrm{a}}}\end{array}$ & None reported \\
\hline \multicolumn{3}{|l|}{ TG synthesis } \\
\hline Depots & $\begin{array}{l}\uparrow \text { TG synthesis in scFEM vs scABD } \\
\text { depots [38] }\end{array}$ & $\begin{array}{l}\uparrow \text { TG storage in scABD depot of } \\
\text { obese vs lean [41] }\end{array}$ \\
\hline Race & $\begin{array}{l}\uparrow \text { TG synthesis in white vs } \\
\text { African-American individuals [38] }\end{array}$ & None reported \\
\hline Insulin sensitivity & $\begin{array}{l}\downarrow \text { TG synthesis in insulin-resistant vs } \\
\text { insulin-sensitive individuals [37] }\end{array}$ & None reported \\
\hline TG turnover & TG half-life $\rightarrow \sim 6-12$ months $[33]$ & $\begin{array}{l}\text { TG age (i.e. lifespan) } \rightarrow \sim 1.6 \\
\quad \text { years [41] }\end{array}$ \\
\hline
\end{tabular}

${ }^{a}$ Data challenge the adipose expandability hypothesis formation and subsequent death over a period of years. Heavy water is suitable for human consumption over a period of weeks or months, allowing the ${ }^{2} \mathrm{H}$ labelling approach to provide short-term measures of adipose turnover during a variety of interventional studies, such as diet, exercise and pharmacological treatments. Using birth-death statistical models, the ${ }^{14} \mathrm{C}$ dating method provided the first estimates of adipocyte lifespan in humans. The ${ }^{2} \mathrm{H}$ labelling protocol has been validated to provide physiological, quantitative measures of in vivo adipose cell formation in humans. However, to date, the ${ }^{2} \mathrm{H}$ approach has not been implemented in the measurement of adipocyte death; therefore, the comprehensive turnover rate of adipocytes has not been reported using this method. Nevertheless, this approach could be used to assess adipocyte death, as the loss of ${ }^{2} \mathrm{H}$ enrichment in the adipose tissue pool after cessation of ${ }^{2} \mathrm{H}_{2} \mathrm{O}$ consumption occurs only by cell loss or death [30].

\section{Conclusions}

Given the strong association between adipose tissue expandability and turnover with metabolic health, further investigations are necessary to enhance our understanding of the dynamics of adipose kinetics in humans. Our recent data provide in vivo evidence that is contrary to the adipose expandability hypothesis [35]; however, it is important to note that these analyses provide cross-sectional data and do not reflect causality. To date, there have been no experimental overfeeding studies to truly examine periods of fat expansion in vivo, as individuals with various capacities for adipose expandability may respond differently. Hence, it remains to be seen how extended periods of weight gain and frequent weight cycling in adulthood affect changes in total adipocyte number and turnover and influence energy metabolism. Interestingly, a recently published study by Arner et al [42] demonstrated that lipolysis is an important predictor of future weight gain and impaired glucose metabolism. Hence, similar prospective studies using an in vivo approach (i.e. ${ }^{2} \mathrm{H}$ labelling) are necessary to clarify the role of adipose turnover in the pathogenesis of insulin resistance and type 2 diabetes. Such in vivo assessments of WAT will yield new knowledge of the dynamics of adipose growth during metabolic diseases, and are likely to direct the discovery of novel therapeutic targets centred on adipocyte generation and death in humans.

Funding Work in the authors' laboratory is partially supported by R01DK090607 (ER) and by a Nutrition Obesity Research Center (NORC) Grant P30DK072476 (ER) through the National Institutes of Health. Work in the authors' laboratory is also supported by K01DK100527 (UW) and R03DK112006 (UW) from the National Institute of Diabetes and Digestive and Kidney Diseases of the National Institutes of Health, which are funding sources for UW.

Duality of interest The authors declare that there is no duality of interest associated with this manuscript. 
Contribution statement Both authors were responsible for drafting the article and revising it critically for important intellectual content. Both authors approved the version to be published.

\section{References}

1. Cinti S (2012) The adipose organ at a glance. Dis Model Mech 5: 588-594

2. Manolopoulos KN, Karpe F, Frayn KN (2010) Gluteofemoral body fat as a determinant of metabolic health. Int J Obes 34:949-959

3. Vague J (1956) The degree of masculine differentiation of obesities: a factor determining predisposition to diabetes, atherosclerosis, gout, and uric calculous disease. Am J Clin Nutr 4:20-34

4. Lemieux S, Prud homme D, Bouchard C, Tremblay A, Despres JP (1993) Sex differences in the relation of visceral adipose tissue accumulation to total body fatness. Am J Clin Nutr 58:463-467

5. Karastergiou K, Fried SK (2012) Sex differences in human adipose tissues - the biology of pear shape. Biol Sex Differ 3:13

6. Karpe F, Pinnick KE (2015) Biology of upper-body and lower-body adipose tissue-link to whole-body phenotypes. Nat Rev Endocrinol 11:90-100

7. Lundgren M, Svensson M, Lindmark S, Renstrom F, Ruge T, Eriksson JW (2007) Fat cell enlargement is an independent marker of insulin resistance and 'hyperleptinaemia'. Diabetologia 50:625633

8. Michaud A, Laforest S, Pelletier M et al (2016) Abdominal adipocyte populations in women with visceral obesity. European J Endocrinol 174:227-239

9. Lonn M, Mehlig K, Bengtsson C, Lissner L (2010) Adipocyte size predicts incidence of type 2 diabetes in women. FASEB J 24:326331

10. Weyer C, Foley JE, Bogardus C, Tataranni PA, Pratley RE (2000) Enlarged subcutaneous abdominal adipocyte size, but not obesity itself, predicts type II diabetes independent of insulin resistance. Diabetologia 43:1498-1506

11. Danforth E Jr (2000) Failure of adipocyte differentiation causes type II diabetes mellitus? Nat Genet 26:13

12. Arner E, Westermark PO, Spalding KL et al (2010) Adipocyte turnover: relevance to human adipose tissue morphology. Diabetes 59:105-109

13. Virtue S, Vidal-Puig A (2010) Adipose tissue expandability, lipotoxicity and the metabolic syndrome-an allostatic perspective. Biochim Biophys Acta 1801:338-349

14. McLaughlin T, Sherman A, Tsao P et al (2007) Enhanced proportion of small adipose cells in insulin-resistant vs insulin-sensitive obese individuals implicates impaired adipogenesis. Diabetologia 50:1707-1715

15. Pasarica M, Xie H, Hymel D et al (2009) Lower total adipocyte number but no evidence for small adipocyte depletion in patients with type 2 diabetes. Diabetes Care 32:900-902

16. McLaughlin T, Lamendola C, Coghlan N et al (2014) Subcutaneous adipose cell size and distribution: relationship to insulin resistance and body fat. Obesity (Silver Spring) 22:673-680

17. Johannsen DL, Tchoukalova Y, Tam CS et al (2014) Effect of 8 weeks of overfeeding on ectopic fat deposition and insulin sensitivity: testing the 'adipose tissue expandability hypothesis. Diabetes Care 37:2789-2797

18. McLaughlin T, Craig C, Liu LF et al (2016) Adipose cell size and regional fat deposition as predictors of metabolic response to overfeeding in insulin-resistant and insulin-sensitive humans. Diabetes 65:1245-1254

19. Hauner H, Entenmann G (1991) Regional variation of adipose differentiation in cultured stromal-vascular cells from the abdominal and femoral adipose tissue of obese women. Int J Obes 15:121-126
20. Tchoukalova YD, Koutsari C, Votruba SB et al (2010) Sex- and depot-dependent differences in adipogenesis in normal-weight humans. Obesity (Silver Spring) 18:1875-1880

21. Tchkonia T, Giorgadze N, Pirtskhalava T et al (2002) Fat depot origin affects adipogenesis in primary cultured and cloned human preadipocytes. Am J Physiol Regul Integr Comp Physiol 282: R1286-R1296

22. Tchoukalova YD, Votruba SB, Tchkonia T, Giorgadze N, Kirkland JL, Jensen MD (2010) Regional differences in cellular mechanisms of adipose tissue gain with overfeeding. Proc Natl Acad Sci U S A 107:18226-18231

23. Krotkiewski M, Bjorntorp P, Sjostrom L, Smith U (1983) Impact of obesity on metabolism in men and women. Importance of regional adipose tissue distribution. J Clin Invest 72:1150-1162

24. Tchoukalova YD, Koutsari C, Karpyak MV, Votruba SB, Wendland E, Jensen MD (2008) Subcutaneous adipocyte size and body fat distribution. Am J Clin Nutr 87:56-63

25. Cinti S, Mitchell G, Barbatelli G et al (2005) Adipocyte death defines macrophage localization and function in adipose tissue of obese mice and humans. J Lipid Res 46:2347-2355

26. MacKellar J, Cushman SW, Periwal V (2010) Waves of adipose tissue growth in the genetically obese Zucker fatty rat. PLoS One 5: e8197

27. Niesler CU, Siddle K, Prins JB (1998) Human preadipocytes display a depot-specific susceptibility to apoptosis. Diabetes $47: 1365-$ 1368

28. Papineau D, Gagnon A, Sorisky A (2003) Apoptosis of human abdominal preadipocytes before and after differentiation into adipocytes in culture. Metabolism 52:987-992

29. Salans LB, Cushman SW, Weismann RE (1973) Studies of human adipose tissue. Adipose cell size and number in nonobese and obese patients. J Clin Invest 52:929-941

30. Busch R, Neese RA, Awada M, Hayes GM, Hellerstein MK (2007) Measurement of cell proliferation by heavy water labeling. Nat Protoc 2:3045-3057

31. Neese RA, Misell LM, Turner S et al (2002) Measurement in vivo of proliferation rates of slow turnover cells by $2 \mathrm{H} 2 \mathrm{O}$ labeling of the deoxyribose moiety of DNA. Proc Natl Acad Sci U S A 99:1534515350

32. Turner SM, Murphy EJ, Neese RA et al (2003) Measurement of TG synthesis and turnover in vivo by ${ }^{2} \mathrm{H}_{2} \mathrm{O}$ incorporation into the glycerol moiety and application of MIDA. Am J Physiol Endocrinol Metab 285:E790-E803

33. Strawford A, Antelo F, Christiansen M, Hellerstein MK (2004) Adipose tissue triglyceride turnover, de novo lipogenesis, and cell proliferation in humans measured with ${ }^{2} \mathrm{H}_{2} \mathrm{O}$. Am J Physiol Endocrinol Metab 286:E577-E588

34. White UA, Fitch MD, Beyl RA, Hellerstein MK, Ravussin E (2016) Differences in in vivo cellular kinetics in abdominal and femoral subcutaneous adipose tissue in women. Diabetes 65: 1642-1647

35. White UA, Fitch MD, Beyl RA, Hellerstein MK, Ravussin E (2017) Association of in vivo adipose tissue cellular kinetics with markers of metabolic health in humans. J Clin Endocrinol Metab 102:2171-2178

36. Hirsch J, Fried SK, Edens NK, Leibel RL (1989) The fat cell. Med Clin North Am 73:83-96

37. Allister CA, Liu LF, Lamendola CA et al (2015) In vivo ${ }^{2} \mathrm{H}_{2} \mathrm{O}$ administration reveals impaired triglyceride storage in adipose tissue of insulin-resistant humans. J Lipid Res 56: 435-439

38. White UA, Fitch MD, Beyl RA, Hellerstein MK, Ravussin E (2018) Racial differences in in vivo adipose lipid kinetics in humans. J Lipid Res 59:1738-1744 
39. Carnethon MR, Palaniappan LP, Burchfiel CM, Brancati FL, Fortmann SP (2002) Serum insulin, obesity, and the incidence of type 2 diabetes in black and white adults: the atherosclerosis risk in communities study: 1987-1998. Diabetes Care 25:13581364

40. Spalding KL, Arner E, Westermark PO et al (2008) Dynamics of fat cell turnover in humans. Nature 453:783-787
41. Arner P, Bernard S, Salehpour M et al (2011) Dynamics of human adipose lipid turnover in health and metabolic disease. Nature 478: $110-113$

42. Arner P, Andersson DP, Backdahl J, Dahlman I, Ryden M (2018) Weight gain and impaired glucose metabolism in women are predicted by inefficient subcutaneous fat cell lipolysis. Cell Metab 28: 45-54:e3 